\title{
MOTOR NEURON DISEASE
}

\section{Functional screening identifies novel candidate risk genes in amyotrophic lateral sclerosis}

\author{
$\mathrm{n}$ a new study, published in PNAS, \\ Couthouis et al. used a yeast \\ functional screen, in combination
} with biochemical, neuronal cell culture, Drosophila and human genetic studies, to identify novel candidate susceptibility genes for amyotrophic lateral sclerosis (ALS). This screening method could help to identify other causative genes for ALS and similar neurodegenerative diseases, and these findings could help to explain the mechanistic basis of these disorders.

ALS is a progressive neurodegenerative disease of the upper and lower motor neurons that causes extensive muscle paralysis. Both familial and sporadic forms of ALS have been reported, and pathogenic mutations have been identified in a number of genes. Two proteins encoded by these genes, TDP-43 and FUS, are RNA-binding proteins that have also been linked to frontotemporal dementia. Aggregates of TDP-43 and FUS have been found in the motor neurons in patients with ALS.

"We have been using a simple yeast model system to define the key features that contribute to the aggregation and toxicity of TDP-43 and FUS and to screen for genetic modifiers of these phenotypes," says Aaron Gitler, from the University of Pennsylvania, PA, USA and lead researcher of the study. "We wondered if other RNA-binding proteins might have similar properties as TDP-43 and FUS and thus be candidate genes for ALS."

To test their hypothesis, Gitler and colleagues cloned 133 of 213 human RNAbinding proteins and expressed them in yeast. Using fluorescence microscopy and spotting assays, they identified 33 of these proteins that aggregated in the cytoplasm and were toxic. "In other words, these proteins behaved in yeast just like the ALS-associated proteins TDP-43 and FUS," explains Gitler.

In addition to an RNA-recognition motif, FUS and TDP-43 also have a prion-like domain, which is critical for the aggregation of these proteins. The authors point out
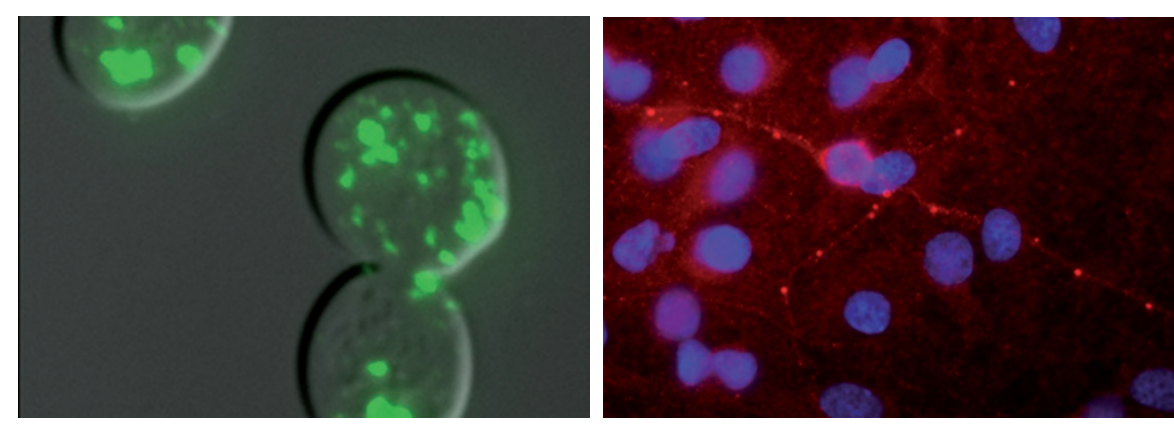

TAF15 forms cytoplasmic aggregates when expressed in yeast (left panel). Mutant TAF15 (red) also forms cytoplasmic aggregates when this protein is expressed in cultured neurons from rat embryos (right panel). Images courtesy of A. Gitler.

that the prion-like domain is a shared feature of many proteins that are involved in neurodegenerative diseases, which may be important, as 'prionoid' aggregation of proteins is often evident in the neurons of patients with these disorders. Using a bioinformatics approach, the investigators found that eight of the 33 toxic RNAbinding proteins had a prion-like domain similar to that in TDP-43 and FUS.

"We then sequenced one of these genes, TAF15, in a large collection of patients with ALS and in healthy controls" says Gitler. "We identified missense variants in the patients with ALS that were not present in the controls." Moreover, examination of postmortem tissue from patients with ALS revealed that TAF15 was misclocalized in the cytoplasm of the motor neurons in some individuals.

Gitler and colleagues also tested the function of TAF15 in animal and insect models. When TAF15 was expressed in motor neurons cultured from rat embryos, the ALS variants of the gene caused cytoplasmic aggregation in the primary motor neurons, whereas expression of the wild-type gene did not. Furthermore, expression of TAF15 in Drosophila led to neurodegeneration and a shortened lifespan. In vitro, the purified wild-type protein was inherently aggregation-prone; however, the ALS-linked variants of TAF15 showed accelerated rates of aggregation.

"While we have not proven that TAF15 is an ALS gene, we have provided multiple lines of compelling experimental evidence that support a role for TAF15 in ALS," asserts Gitler. "We put forth a new concept that multiple RNA-binding proteins, in addition to TDP-43 and FUS, could contribute to ALS and related neurodegenerative diseases."

Gitler and colleagues are currently analyzing whether mutations in any of the other toxic RNA-binding proteins are evident in patients with ALS. "We are beginning to refine the genetic landscape of ALS," says Gitler. "This should help to stratify patient populations based on genetic mutations, which should help in clinical trials."

Couthouis et al. note that next-generation sequencing will eventually become routine in personalized medicine, and will enable identification of all the genetic causes of ALS. Meanwhile, the techniques used by these researchers will provide a basis for identifying novel genetic risk factors for ALS. "Once we know all of the genetic contributors to ALS, we will hopefully be able to develop a deeper mechanistic understanding into disease pathogenesis, which should spur new therapeutic strategies," Gitler concludes.

Katy Malpass

Original article Couthouis, J. et al. A yeast functional screen predicts new candidate ALS disease genes. Proc Natl Acad. Sci. USA doi:10.1073/pnas.1109434108 